American Journal of Infectious Diseases 5 (1): 1-6, 2009

ISSN 1553-6203

(C) 2009 Science Publications

\title{
Incident of Pseudomonas aeruginosa in Post-Operative Wound Infection
}

\author{
${ }^{1}$ Hani A. Masaadeh and ${ }^{2}$ Adnan S. Jaran \\ ${ }^{1}$ Faculty of Medicine, \\ Jordan University of Science and Technology, Irbid, Jordan \\ ${ }^{2}$ Department of Biological Science, \\ Al al-Bayt University, P.O. Box 130040, Mafraq 25113, Jordan
}

\begin{abstract}
The primary aim of this study was to determine the incidents of pseudomonas aeruginosa in post-operative wound infection and its sensitivity pattern to commonly used antibiotics. During a period of six months between February to December, 2005, 115 specimens were collected from King Abdullah University Hospital, Princess Basma Hospital, Princess Badea and Princess Rahma Hospitals. Samples were obtained from the hospitals and processed in our research microbiology lab. Assessment of Pseudomonas aeruginosa as an agent of nosocomial infections. Out of the 115 bacterial isolates found in post-operative wound infection, 20 (27.8\%) were Pseudomonas aeruginosa, followed by E. coli 18 (15.6\%), S. aureus 17 (14.7\%), Acinetobacter calcoaceticus 15 (13.0\%), K. species 14 (12.1\%), Proteus species 7 (6.0\%), Citrobacter freundii 4 (3.4\%), Streptococcus pyogenes 3 (2.6 \%), Enterococcus faecalis $3(2.6 \%)$ and no growth $2(1.7 \%)$. The results showed that the occurrence of Pseudomonas aeruginosa was higher in young groups than in the other groups. The lowest causative agents of post operation infections were Streptococcus pyogenes, Enterococcus faecalis and Citrobacter freundii. There was no significant difference in the occurrence of hospital infection between males and females. The sensitivity pattern of $P$. aeruginosa isolated from patients in postoperative wound infections, the organism was sensitive to amikacin, gentamicin, tobramycin, ciprofloxacin and aztreonam with amikacin showing the highest percentage sensitivity. In conclusion this study shows that there is an increased rate of incidence of Pseudomonas aeruginosa in postoperative wound infections.
\end{abstract}

Key words: Post-operative wound, infection, Pseudomonas aeruginosa, incident, nosocomial, antibiotic

\section{INTRODUCTION}

Post-operative wound infection simply means wound infection after surgical operation, may originate during the operation i.e. as a primary wound infections or may occur after the operation from sources in the ward or as a result of some complications i.e. secondary wound infection ${ }^{[1]}$.

There are multiple reasons for post-operative wound infections, which have been validated and documented as risk factors. A risk factor is any recognized contribution to an increase in post-operative wound infection ${ }^{[1]}$.

The virulence and invasive capability of the organisms have been reported to influence the risk of infection, but the physiological state of the tissue in the wound and immunological integrity of the host seem to be of equal importance in determining whether infection occurs ${ }^{[2]}$.
Primary infections are usually more serious appearing within 5-7 days of operation. Epidemiologically these infections are mostly related to endogenous flora and could be related to a member of the operating team or to some other environmental sources in the operating theater ${ }^{[3]}$.

Maniatis et al. ${ }^{[4]}$ suggested that deep-seated sepsis developing a few days after an operation and before the wound has been dressed reflect a theatre infection.

Studies support the concept that a reduction in post-operative wound infection is directly related to increased education and awareness of its causes, its prevention is greatly aided by critically evaluated infection control practice ${ }^{[5]}$.

The incidence of primary wound infection is correlated to the bacteriological cleanliness of the operation. Clean operation $(<2 \%)$ dose not involve opening a viscous or cutting a cross mucus membranes

Corresponding Author: Adnan S. Jaran, Department of Biological Science, Al al-Bayt University, P.O. Box 130040, Mafraq 25113, Jordan. Tel: 962-2-6297000/Ex 2119 Fax 962-2-6297034 
normally colonized with bacterial flora. In contaminated operations $(20 \%)$, a viscous normally containing bacteria or a membrane normally colonized with bacteria is incised, while in clean-contaminated operations $(<10 \%)$ a viscous or membrane which is usually sterile $e^{[5]}$.

Ward infections tend to be more superficial and frequently follow the dressing of wounds in the ward. Similarly, skin infection such as boils or abscesses developing at sites other than the operation site indicate that the infection was acquired in the $\operatorname{ward}^{[2,4]}$. Wound infection after contaminated operations are usually caused by the bacteria normally resident in the opened viscous or on the incised mucus membrane, i.e. the bacteria belong to the patient's own normal flora, or that which has been gained while he/she is in a hospital ${ }^{[6,7]}$. Combined with a regular feed back to the surgeon it has been shown to decrease the infection rate. It is important to realize that surveillance alone cannot act as a substitute for action and prevention ${ }^{[2]}$.

Operations which are carried out through a field already contaminated by bacteria. Such as abscesses and colon operation ${ }^{[8,9]}$. Bacteriological studies have shown that post-operative wound infection is universal and that the bacteria types present vary with geographical location, bacteria resident on the skin, clothing at the site of wound, time between wound and examination ${ }^{[10,11]}$. Facultative aerobic Gram-negative bacilli, streptococci and staphylococci remain in the colon, regardless of the type of preparation. The bowel and post-operative infection in colon rectal surgery without systemic intra-operative prophylaxis can be as high as $50 \%^{[12]}$. Within recent years, there has been a growing prevalence of Gram-negative organisms as causes of serious infections seen in many hospitals. These organisms have almost replaced Staphylococcus aureus in nosocomial infection. Of the Gram-negative bacilli, Pseudomonas aeruginosa has been of particular interest ${ }^{[13]}$.

After contaminated operations infections rates are more variable, depending on the type and number of organisms released from the membrane. An empty stomach is normally sterile but bacterial counts in the stomach can reach more than $10^{6} \mathrm{CFU} / \mathrm{ml}$ during gastric bleeding. Bacteria of the normal flora of the upper respiratory tract and Gram-negative bacilli are able to survive in the stomach after swallowing ${ }^{[14]}$.

The incidence of Pseudomonas aeruginosa in postoperative wound infection is becoming more serious in developing countries because of relaxation in general hygienic measures, mass production of low quality antiseptic and medicinal solutions for treatment, difficulties in proper definition of the responsibility among the hospital staff ${ }^{[5]}$. Escherichia coli, other Gram-negative bacilli, Enterococci, Beta-hemolytic streptococci and S. aureus are the important causes of infection in biliary surgery, when stones are present, carcinomas of the biliary tract and after emergency operation ${ }^{[16]}$.

Post-operative infections in the vaginal vault after hysterectomy and in hand and neck surgery after incision of the respiratory mucus membrane is also usually caused by organisms of the local flora, such as Gram-positive cocci and Bacteriodes spp. ${ }^{[17]}$. The hospital-acquired nature of infections with Pseudomonas aeruginosa has been noted and while some patients suffer endogenous infections, the vast majority are acquired from exogenous sources. It has also been observed that healthy carriers of Pseudomonas aeruginosa in the hospital environment account for about $28 \%$ while less in the open community $^{[18]}$.

\section{MATERIALS AND METHODS}

Specimen collection: The specimens were collected from patients aseptically with sterile cotton wool swab suffering from post operation wound infection at King Abdullah Hospital (KAUH), Princess Basma Hospital, Princess Badea Hospital and Princess, Rahma Hospital from February to December, 2005.

Culture media and tests: Several media and tests were used for the isolation, identification and testing the susceptibility of the isolates for common used antibiotics. The media used are:

Blood agar (with 5-7\% defibrinized blood), MacConkey agar, chocolate agar, nutrient agar, Mannitol salt agar. Simmons citrate agar, kligler Iron Agar (KIA), Mueller-Hinton agar, Sulfide formation indole production, motility Test (SIM), Nutrient agar, Methyl Red-Voges Proskauer broth, Thioglycollate broth, Coagulase, Catalase, Urease, Oxidase tests were used for the identification. All of the above media and reagents were obtained from (Difco. USA).

The media were prepared according to manufacturers instructions in $500 \mathrm{~mL}$ bottle and sterilized by autoclaving at $121^{\circ} \mathrm{C}$ for $20 \mathrm{~min}$.

All wound swabs collected for bacteriological investigations during the period of this study were treated according to established method of treating wound swabs ${ }^{[9]}$. Gram stain preparations were made from all the swabs The plates were incubated at $37^{\circ} \mathrm{C}$ for 18-24 $\mathrm{h}$ in an incubator. The plates were read the following day but extended to $48 \mathrm{~h}$ if there was no 
bacterial growth within $24 \mathrm{~h}$. Isolated colonies were subjected to Gram staining technique and biochemical tests for identification.

Antibiotic sensitivity tests were carried out on isolated and identified colonies of Pseudomonas aeruginosa using commercially prepared antibiotic sensitivity disc using Kirby-Bauer method. Antibiotic testing was not done on other bacterial isolates in this study since our focus was on the incident of Pseudomonas aeruginosa.

Standard strains of Pseudomonas aeruginosa (NCTC 15442), Staphylococcus aureus (ATCC 6538) and Escherichia coli (NCTC 10561) were used as controls.

\section{RESULTS AND DISCUSSION}

A total of 115 samples were collected from surgical, pediatric, orthopedic, obstetrics and gynecology wards suffering from post operation infection, all specimens were directly transferred to the microbiology lab and cultured to the appropriate media (as descried in materials and methods).

Table 1 shows the occurrence of Pseudomonas aeruginosa in post-operative wound infection in relation to age. The age groups were divided into seven catagories: 10-20, 21-30, 31-40, 41-50, 51-60, 61-70 and 71 and above. The result showed that the occurrence of Pseudomonas aeruginosa was higher in young groups than in the other groups.

Table 2 shows the types and frequency of surgery that have been done for each patient finding that the wound infections and caesarean sections have the most frequency (10 cases for each) and the highest percentage ( $8.6 \%$ for each).

Table 3 shows the most causative agent of post operation infections was $P$. aeruginosa 32 isolates (27.8\%), following E. coli 18 isolates (15.6\%). The lowest causative agents of post operation infections were Streptococcus pyogenes, Enterococcus faecalis and Citrobacter freundii.

\begin{tabular}{llll} 
Table 1: & $\begin{array}{l}\text { Relationship between } \\
\text { relation to age }\end{array}$ & post-operative wound infections in \\
\hline Factors & No. samples & No. P. aeruginosa & P. aerioginosa $(\%)$ \\
\hline $10-20$ & 8 & 3 & 9.0 \\
$21-30$ & 10 & 6 & 18 \\
$31-40$ & 25 & 7 & 21 \\
$41-50$ & 17 & 4 & 12.5 \\
$51-60$ & 18 & 5 & 15.6 \\
$61-70$ & 25 & 4 & 12.5 \\
$71-$ above & 12 & 3 & 9.0 \\
\end{tabular}

Table 4 shows the relationship between postoperative wound infections and duration of operation, most of the surgeries took $1.0 \mathrm{~h} 42$ (36.9\%), which indicates that the majority of the surgical procedures were minor surgeries.

Table 5 shows the relationship between postoperative wound infections and sex. There was no significant difference in the occurrence of hospital infection between males and females.

Table 6 shows Sensitivity pattern of $P$. aeruginosa isolated from patients in post-operative wound infections, the organism was sensitive to amikacin, gentamicin, tobramycin, ciprofloxacin and aztreonam, with amikacin showing the highest percentage sensitivity.

Table 2: The types and frequency of surgeries

\begin{tabular}{lll}
\hline Type of surgery & Frequency of surgery & (\%) \\
\hline Abdominal abscess & 6 & 5.2 \\
Abscess drainage & 4 & 3.4 \\
Bone excision & 4 & 3.4 \\
Caecerean section & 10 & 8.6 \\
Diabetic foot & 9 & 7.8 \\
Fasciotomy & 4 & 3.4 \\
Herinorhaphy & 2 & 1.7 \\
HIP abscess & 5 & 4.3 \\
Labyriulhectomy & 4 & 3.4 \\
Lipoma excision & 3 & 2.6 \\
Liver abscess & 2 & 1.7 \\
Lumbarectomy & 2 & 1.7 \\
Mastiodectomy & 9 & 7.8 \\
Nail removal & 4 & 3.4 \\
Neck abscess & 9 & 7.8 \\
Open heart & 5 & 4.3 \\
Open knee wound & 4 & 3.4 \\
Perianal fistula & 4 & 3.4 \\
Removal of metal & 4 & 3.4 \\
Septoplasty & 2 & 1.7 \\
Skin graft & 4 & 3.4 \\
Thoracotomy & 2 & 1.7 \\
Thyroidectomy & 3 & 2.6 \\
Wound abscess & 10 & 8.6 \\
Total & 115 & 100 \\
\hline
\end{tabular}

Table 3: Frequency and percentage of microorganisms isolated from patients in post-operative wound infection

\begin{tabular}{lll}
\hline Microorganism & Number of isolated & $\mathbf{( \% )}$ \\
\hline P. aeruginosa & 32 & 27.8 \\
E. coli & 18 & 15.6 \\
S. aureus & 17 & 14.7 \\
Acinetobacter calcoaceticus & 15 & 13.0 \\
K. species & 14 & 12.1 \\
Proteus species & 7 & 6.0 \\
Citrobacter freundii & 4 & 3.4 \\
Streptococcus pyogenes & 3 & 2.6 \\
Enterococcus faecalis & 3 & 2.6 \\
No growth & 2 & 1.7 \\
Total & 115 & 100 \\
\hline
\end{tabular}


Am. J. Infect. Dis., 5 (1): 1-6, 2009

Table 4: Relationship between postoperative wound infections and duration of operation

\begin{tabular}{lll}
\hline Duration of surgery $\mathrm{h}^{-1}$ & Number of surgery & $(\%)$ \\
\hline 0.5 & 8 & 6.9 \\
1.0 & 42 & 36.5 \\
1.5 & 9 & 7.8 \\
2.0 & 10 & 8.6 \\
2.5 & 4 & 3.4 \\
3.0 & 10 & 8.6 \\
3.5 & 5 & 4.3 \\
4.0 & 11 & 9.5 \\
4.5 & 3 & 2.6 \\
5.0 & 2 & 1.7 \\
5.5 & 3 & 2.6 \\
6.0 & 3 & 2.6 \\
7.0 & 2 & 1.7 \\
8.0 & 3 & 2.6 \\
Total & 115 & 100 \\
\hline
\end{tabular}

Table 5: Relationship between postoperative wound infections and sex

\begin{tabular}{ll}
\hline Sex & Total \\
\hline Male & $60(52.2 \%)$ \\
Female & $55(47.8 \%)$ \\
Total & $115(100 \%)$ \\
\hline
\end{tabular}

Table 6: Sensitivity pattern of $P$. aeruginosa isolated from patients in post-operative wound infection $(\mathrm{n}=32)$

\begin{tabular}{ll}
\hline Antibiotics & Sensitive (\%) \\
\hline Amikacin & 78 \\
Gentamicin & 72 \\
Tobramycin & 69 \\
Ciprofloxacin & 66 \\
Aztronam & 58 \\
Ceftazidime & 44 \\
Piperacilin & 19 \\
Meropenim & 16 \\
Cefepime & 9 \\
Tetracycline & 6 \\
\hline
\end{tabular}

The primary aim of this study was to determine the incident of Pseudomonas aeruginosa in post-operative wound infection and its sensitivity pattern to commonly used antibiotics.

During a period of six months between February to December, 2005, 115 specimens were collected from King Abdullah University Hospital, Princess Basma Hospital, Princess Badea Hospital and Princess, Rahma Hospital (local hospitals) (Table 5), suffering from post operation infection, all specimens were directly transferred to the lab and cultured to the appropriate media (as descried in materials and methods).

Hospital acquired infection is a serious problem of most hospitals. Infection may be acquired during the operation or postoperatively in wards ${ }^{[1]}$. The results obtained showed a high incidence of Pseudomonas $(27.8 \%)$ of all the pathogens isolated from the postoperative wound in the different operations.
Bertrand et al. ${ }^{[15]}$ recorded prevalent rate of $19.1 \%$. The result of our study is higher than that reported by ${ }^{[15]}$. This could be attributed to differences in geographical location and hygienic measures.

The results of the study shows that the overall infection rate was slightly influenced by age and the general health of the patients and these results are in agreement with the observation by ${ }^{[19]}$, in which the overall rate of infection was influenced by the age and health of the patients. Since the majority of the population in our study was within the same age that has been reported in the literature. 55 of our patients $(83.3 \%)$ were over 28 years of age. MCEwIN ${ }^{[20]}$ reported that postoperative infection increases with the period of time over which the patient has been in hospital before operation, presumably exposed to skin colonization by "hospital" bacteria.

In the present study, data showed that, there was no difference between sexes with regard to the susceptibility to infection (Table 5). This is not consistent with the previous finding ${ }^{[21]}$.

Since, the percentage of wound infection increased as the duration of operation increased, from $43 \%$ at less than $60 \mathrm{~min}$, up to $57 \%$ at more than $90 \mathrm{~min}$ intervals (Table 4). This means that chances of exposure to bacteria was high, which increased the overall rate of postoperative wound infection.

This also revealed the increasing incidence of Pseudomonas aeruginosa in post-operative wound infections as observed by other scientists especially in recent years. Joshi et al. ${ }^{[15]}$ quoting Najak stated that Pseudomonas aeruginosa has almost replaced Staphylococcus aureus in post-operative wound infection and reported that Najak documented 16.8\% for Pseudomonas aeruginosa and 5.6\% for Staphylococcus aureus. It is thus clear that the prevalent rate of Pseudomonas aeruginosa recorded in this study is in agreement with that obtained in other hospitals.

The susceptibility pattern of the 32 isolates of Pseudomonas aeruginosa to some commonly used antibiotics as reported in this study is similar to that found reported in the literature ${ }^{[22]}$.

Hasseigren et al. ${ }^{[23]}$ studied 112 patients to find the sources of bacteria causing wound infection. They found that the patients were the sources of bacteria in all cases of wound infection. The increase in postoperative infections was due to high penicillinresistant carrier rate in hospital personnel and patients as a result of widespread use of Penicillin ${ }^{[23]}$. However, Krieger et al. ${ }^{[21]}$ reported that the scrubbed 
team and patients were the major source of wound contamination.

\section{CONCLUSION}

This study shows that there is an increased rate of incidence of Pseudomonas aeruginosa in post-operative wound infections. The most causative agent of post operation infections were $P$. aeruginosa, followed by $E$. coli, S. aureus, Acinetobacter calcoaceticus and Klebsiella spieces. The lowest causative agents of post operation infections were, Streptococcus pyogenes, Enterococcus faecalis and Citrobacter frenudii. This is in agreement with survey studies carried out in various hospitals.

The infection appears to be common in hospitals with relaxed hygienic measures and is dependent on age, sex and duration of stay in the hospital. The reason for this increase in postoperative infection rate with prolonged preoperative hospitalization is primarily due to colonization of patients with hospital-acquired resistant microorganisms.

\section{ACKNOWLEDGEMENT}

We are grateful to the staff of all primary health care centers and all patients included in this study for their support and assistance.

\section{REFERENCES}

1. Mousa, H., 1997. Aerobic, anaerobic and fungal burn wound infections. J. Hosp. Infect., 37: 317-323. Doi doi:10.1016/S0195-6701(97) 90148-1.

2. Heinzelmann, M., M. Scott and T. Lam, 2002. Factors predisposing to bacterial invasion and infection. Am. J. Surgery, 183: 179-190. http://general.surgery.duke.edu/wysiwyg/download s/InfectionFactors.pdf

3. Ayliffe, G.A.J., J.R. Babb and L.J. Taylor, 1999. The Hospital Environment. In: Hospital-Acquired Infection. Principles and prevention. 3rd Edn. pp: 109-121. Butterworth-Heinemann, Oxford. ISBN: 0750621052.

4. Maniatis, A.N., I.P. Trougakos, J. Palermos, N.A. Maniatis and N.J. Legakis, 1997. Changing patterns of bacterial nosocomial infections: A nineyear study in a general hospital. Chemotherapy, 43: 69-76. http://www.ncbi.nlm.nih.gov/pubmed/ 8996745 .

5. Leigh, D., F. Emmanuel, Sedgwick and R. Dean, 1990. Post-operative urinary tract infection and wound infection in women undergoing caesarean section: A comparison of two study periods in 1985 and 1987 J. Hosp. Infect., 15: 107-116. http://www.ncbi.nlm.nih.gov/pubmed/1969432
6. Andenaes, K., E. Lingaas, P.F. Amland, K.E. Giercksky and F. Abyholn, 1996. Preoperative bacterial colonization and its influence on post operative wound infection in plastic surgery. J. Hosp. Infect., 34(4): 291-299. doi:10.1016/S0195-6701(96)90109-7.

7. Zerahn, B., M. storgaad and M. Arendrup, 1997. Deep wound infections with salmonella enteritides following osteosynthesis. Ugeskr Laagr, 160: 53-55. http://www.ncbi.nlm.nih.gov/pubmed/ 9446267.

8. Russell, R.C.G., N.S. Williams and C.J.K. Bulstrode, 2000. Bailey and Love's Short Practice of Surgery. 23rd Edn. Oxford Press. pp: 87-98. ISBN: 0340759240

9. Joffe, S.N., W.O. Thomson, J. McGavigan and P.C. Trexler, 1978. A closed system surgical isolator for major elective abdominal operations. World J. Surgery, 2: 123-127. Doi: 10.1007/BF01574479.

10. Trilla, A., 1994. Epidemiology of nosocomial infections in adult intensive care units. Intensive Care Med., 20: 1-4. Doi: 10.1007/BF01745243.

11. Anupurba, S., A. Bhattacharjee, A. Garg and M.R. Sen, 2006. Antimicrobial susceptibility of Pseudomonas aeruginosa isolated from wound infections. Indian J. Dermatol., 51: 286-288. http://www.e-ijd.org/text.asp?2006/51/4/286/30298

12. Mehta, G., S. Khanna, B. Trehan and V. Gupta, 1990. Postoperative infection in cardiac surgery: The influence of a change in prophylactic antibiotic regimen. J. Hosp. Infect., 15: 353-362. http://www.ncbi.nlm.nih.gov/pubmed/1972951.

13. Andreassen, J.J., B. Korsager, P. Alstrup and O.B. Jepsen, 2002. Post-operative wound infection: Indicator of clinical quality? Dan. Med. Bull., 49: 242-244. http://www.ncbi.nlm.nih.gov/ pubmed/12238284.

14. Wenzel, R.P. and T.M. Perl, 1995. The significance of nasal carriage of staph aureus and the incidence of postoperative wound infection. J. Hosp. Infect., 31: 13-24. Doi: 10.1016/01956701(95)90079-9.

15. Bertrand, X., M. Thouverez, C. Patry, P. Balvay and D. Talon, 2002. Pseudomonas aeruginosa: antibiotic susceptibility and genotypic characterization of strains isolated in the intensive care unit. Clin. Microbiol. Infect., 7: 706-708. Doi: 10.1046/j.1198-743x.2001.00345.x.

16. Lewis, C.M. and M.J. Zervos, 1990. Clinical manifestations of enterococcal infection. Eur. Clin. Microbial. Infec. Dis., 9: 111-117. doi: 10.1007/BF01963635. 
17. Maurer, I.M., 1978. Hospital Hygiene. 3rd Edn. Edward Arnold Ltd., Colchester, London, pp: 117-127. ISBN: 0713143193.

18. Kolmos, H.J., R.N. Svendsen and S.V. Nielsen, 1997. The surgical team as a source of postoperative wound infections. J. Hosp. Infect., 35: 207-214. Doi: 10.1016/S0195-6701(97)902085.

19. Sangrasi, A.K., A.A. Leghari, A. Aisha Memon, A.K. Talpur, G.A. Qureshi and J.M. Memon, 2008. Surgical site infection rate and associated risk factors in elective general surgery at a public sector medical university in Pakistan. Int. Wound J., 5: 74-78. Doi: 10.1111/j.1742-481X.2007.00365.x.

20. McEwin, R., 2008. The relation between preoperative stay in hospital and postoperative infection. ANZ J. Surgery, 40: 191-194. Doi: 10.1111/j.1445-2197.1970.tb04057.x.
21. Krieger, J.N., D.L. Kaiser and R.P. Wenzel, 1983. Nosocomial urinary tract infections cause wound infections postoperatively in surgical patients. Surg. Gynecol. Obstet., 56: 313-318. http://www.ncbi.nlm.nih.gov/pubmed/6828975.

22. Oluwafemi, O., Oguntibeju and R.A.U. Nwobu, 2004. Occurrence of pseudomonas aeruginosa in post-operative wound infection. Pak. J. Med. Sci., 20: 187-191. http://www.cababstractsplus.org/ google/abstract.asp?AcNo=20043169666.

23. Hasseigren, P.O., A. Saljo, J. Fornander, S. Lundstam, T. Saretok and T. Seeman, 1980. Postoperative wound infections-A prospective study in a newly opened hospital. Ann. Chir. Gynaecol., 69: 269-272. http://www.ncbi.nlm.nih. gov/pubmed/7212602. 